\title{
Fact and fantasy: The effects of internally generated events on the apparent frequency of externally generated events
}

\author{
MARCIA K. JOHNSON and THOMAS H. TAYLOR \\ State University of New York, Stony Brook, New York 11794 \\ and \\ CAROL L. RAYE \\ Barnard College, New York, New York 10027
}

\begin{abstract}
As John Locke pointed out, there are at least two sources of the contents of the mind: events that occur in the world and events that originate in the mind. Memory, as a record of experience, should contain information from both sources. The present studies investigated memory for the frequency of externally and internally generated events. Individual items were presented to subjects varying numbers of times and tested varying numbers of times. Later, subjects were asked to estimate the frequency of both types of events. Experiment 1 showed that internally generated events influenced the judged frequency of externally generated events and vice versa. The first of these was called the IFE effect and the second the IFI effect. Experiment 2 indicated that the IFE effect was greater when tests consisted of overt, as compared to covert, recall trials. The results were discussed in terms of a model for storing and using occurrence information which would account for both our ability to discriminate between and our tendency to confuse internally and externally generated memory representations.
\end{abstract}

Memory research has almost exclusively been concerned with memory for external events (or perceptions), but internally generated events also produce memory traces. Is there confusion between memories for previous experiences and memories for previous imaginations (Johnson, Note 1)? Intuition and the current zeitgeist suggest that there should be. But total confusion of externally and internally generated memories would lead to an inordinate number of false beliefs or delusions, and our memories would not have the minimum degree of accuracy necessary for us to function in the world. How do the representations for externally and internally generated events differ and under what conditions are they confused?

The present experiments are an initial attempt to develop a paradigm for exploring the relationship between memories for external and internal events. It was reasoned that thoughts are tied to perceptual experience in varying degrees. Certainly every whimsical idea does not intermingle indiscriminately with stored perceptions. Yet, if anything is confused with memories for perceptions, it may be mental "regenerations" of perceptual experience, since these perhaps most closely resemble the initial representations of external events.

We would like to acknowledge the very valuable assistance of Roseanne DeVita in conducting these studies. Requests for reprints should be sent to Marcia K. Johnson, Department of Psychology, State University of New York, Stony Brook, New York 11794.
As an index of confusion, the judged frequency of events was chosen, not only because it seemed to be a relatively straightforward task, but also because frequency information has long been an important theoretical construct. Thus, the present experiments were an attempt to determine whether mental regenerations of external events would increase the apparent frequency of occurrence of the events.

\section{EXPERIMENT 1}

The purpose of Experiment 1 was to replicate previous results (e.g., Hintzman, 1969; Howell, 1973; Underwood, Zimmerman, \& Freund, 1971) indicating subjects' ability to judge the relative frequency of occurrence of events, to determine whether people could also judge the relative frequency of internally generated events, and, more importantly, to determine whether the apparent frequency of external events is affected by internally generated events, and vice versa. The basic strategy of this and the following experiment involved manipulating the number of times subjects saw various words and manipulating the number of occasions on which they generated the words. Subsequently, subjects were asked to make frequency judgments.

\section{Method}

Design. Subjects studied and recalled common English words. Within subjects, three levels of study frequency were combined 
factorially with three levels of test frequency. Subjects were later presented with each of the words and half were asked to estimate study frequency and half were asked to estimate test frequency.

Materials. Thirty-six items were formed by using the names, or shorter paraphrases, of 36 Battig and Montague (1969) categories as cues and one high-frequency instance from each category as to-be-remembered items. On a study trial, 18 cueitem pairs were presented and on any given test trial, 18 cues were presented; the subject's task was to write down the appropriate item in the blank beside each cue. Study and test trials alternated for a total of 10 cycles, presented via mimeographed booklets. Individual items were studied 2, 5, or 8 times and were tested 2,5 , or 8 times. An equal number of items were assigned to each of the nine study-test combinations. The sequence of studying and testing items was random, except that no item was tested before it had been studied. Therefore, except for the first trial, not all items on a study trial necessarily appeared on the following test trial. Four random assignments of items to study-test frequencies resulted in four sets of booklets. An equal number of subjects were assigned to each booklet.

Five random orders of all 36 items (without cues) were recorded on tape, at a $5-\mathrm{sec}$ rate, to be used in the frequency judgment task. Within each judgment condition, subjects were assigned randomly to one of these orders.

Procedure. Subjects participated in groups of up to four Instructions to the subjects indicated that their task was to learn the items, so that when a cue was presented they could give the appropriate item; they were not warned that there would be a frequency judgment task. On study trials, $36 \mathrm{sec}$ was allowed ( $2 \mathrm{sec} / \mathrm{item})$, and, on test trials, $54 \mathrm{sec}$ was allowed ( $3 \mathrm{sec} /$ item). Subjects were instructed to read items in order on both study and test trials and not to go back to any item. The experimenter signaled the turning of booklet pages.

After the 10 study-test cycles, subjects were given frequency judgment instructions. Subjects judging the study frequency of items were asked to estimate the number of times (using the numbers $0-10$ ) they had been given an opportunity to study each of the words. Subjects judging the test frequency of items were asked to estimate the number of times (from 0 to 10) they had been given an opportunity to recall each of the words.

Subjects. Twenty introductory psychology students were assigned randomly to each of the two judgment conditions

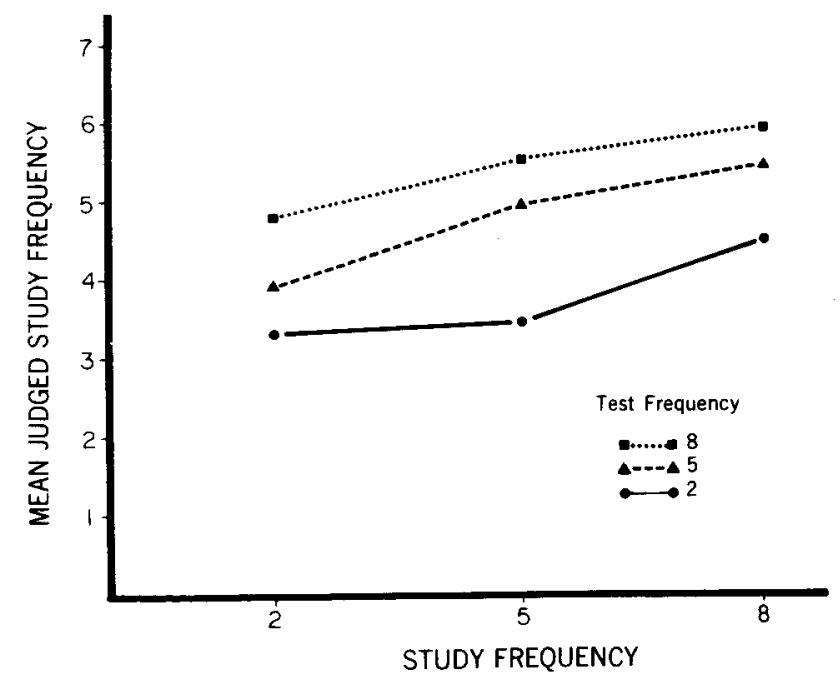

Figure 1. Judged study frequency as a function of manipulated study frequency. Each line represents a different test frequency (Experiment 1).

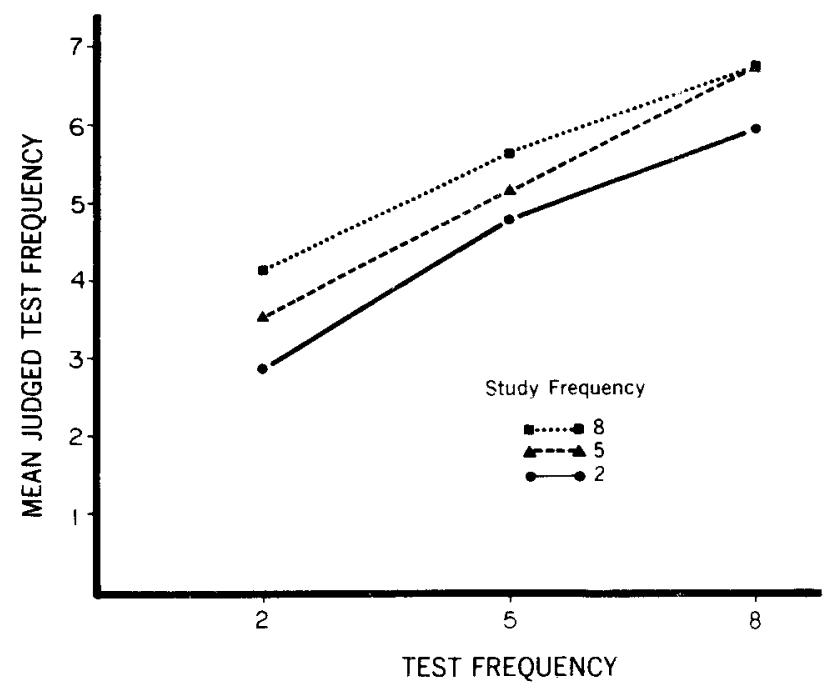

Figure 2. Judged test frequency as a function of manipulated test frequency. Each line represents a different study frequency (Experiment 1).

\section{Results}

Within each judgment condition, each subject contributed four data points to each of the nine cells (representing combinations of study and test frequency) of the design. The four judgments were averaged for each subject and the scores entered into the analyses. Unless otherwise indicated, comparisons were significant at the .05 level or better.

Judgments of study frequency. The data for those subjects asked to judge the number of opportunities they had to study each item are presented in Figure 1. As is apparent from Figure 1, the mean judged frequency of occurrence of items increased as the actual frequency of occurrence increased $[F(2,38)=23.44$, $\mathrm{MSe}=1.09]$. This effect replicates previous findings and indicates that subjects are sensitive to the relative frequency of occurrence of events in the context of the present procedure. As can also be seen in Figure 1, increases in the number of times subjects produced the items resulted in increases in the apparent frequency of occurrence of the items $[F(2,38)=28.36$, MSe $=1.44]$. Although there was also a significant Study Frequency by Test Frequency interaction $[F(4,76)=3.22$, MSe $=.61]$, it does not substantially affect the general form of the main effects.

Judgments of test frequency. The mean scores of those subjects asked to estimate the number of times they were tested on each item are shown in Figure 2. Estimates of test frequency increased with actual increases in test frequency $[\mathrm{F}(2,38)=127.03$, $\mathrm{MSe}=1.04]$, indicating that subjects are sensitive to the relative frequency of internally generated events. In addition, increases in study opportunities contributed to the apparent frequency of tests $[F(2,38)=20.72$, $\mathrm{MSe}=.71]$. 
Comparison of the two types of judgments. The left side of Figure 3 allows a comparison of judged study and judged test frequency as a function of study frequency; the right side shows the two judgments as a function of test frequency. As suggested in Figure 3, there was no type of Judgment by Study Frequency interaction $[\mathrm{F}(2,76)=1.08, \mathrm{MSe}=.90, \mathrm{p}>.25]$, indicating that both types of judgment were equally sensitive to changes in the number of study opportunities. On the other hand, there was a type of Judgment by Test Frequency interaction $[\mathrm{F}(2,76)=10.41, \mathrm{MSe}=1.21]$. As shown in Figure 3 , the judgments of test frequency were more affected by changes in actual test frequency than were the estimates of study frequency. This interaction helps verify that the subjects were attempting to estimate the frequency of occurrence of two different types of events under the two judgment conditions.

The separation between the lines in Figure 1 indicates the extent to which internally generated events Increased the apparent Frequency of Externally generated events (hereafter called the IFE effect); the separation between the lines in Figure 2 indicates the extent to which externally generated events Increased the apparent Frequency of Internally generated events (hereafter called the IFI effect). Although it appears from these graphs that the IFE effect was greater than the IFI effect, this comparison is not reported. More time per item was allowed on test trials than on study trials, under the assumption that it would take more time to generate an item than to read an item, and it is possible that the numbers of functional inputs and outputs differed. Perhaps more importantly, since subjects were forced to respond to each item on test but not on study trials, it could be argued that greater control was exerted over output than over input frequencies. Firmer conclusions regarding the relative magnitudes of the

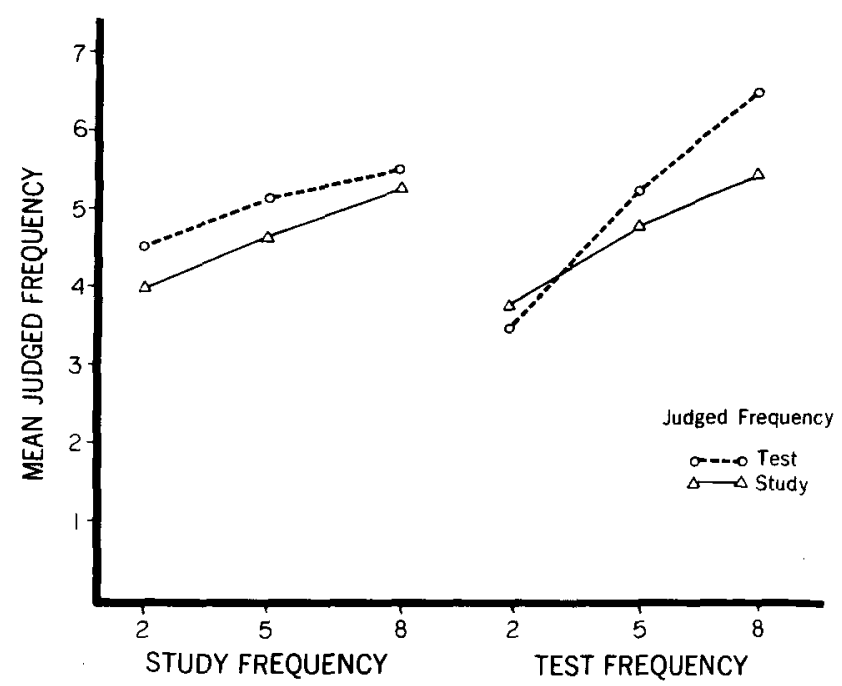

Figure 3. Judged study and test frequencies as a function of study frequency (left panel) and test frequency (right panel) (Experiment 1).
IFE and IFI effects and the relative discriminability among external and internal events would be provided by a study in which rate of presentations of study and test items was systematically varied, and/or in which subjects were forced to respond to each item on both study and test trials.

The present results indicate that subjects are sensitive to the frequency of occurrence of internally generated as well as externally generated events. In addition, they provide direct evidence for confusion between the two types of events. Judgments of the frequency of each type of event were influenced by the frequency of occurrence of the other event.

\section{EXPERIMENT 2}

The test trials in Experiment 1 included recalling and writing down the items. When an item is written down, it is available as new "input" into the system, and, although such inputs are initiated internally, one could argue that they have the status of "external" occurrences. Therefore, Experiment 2 included a comparison of the effects of various types of regenerations on judgments of the frequency of occurrence of external events. The experimental conditions included the previous "standard recall" condition and a new "covert recall" condition, in which subjects indicated whether they could recall the correct responses, but did not write them down. If the covert recall group also showed an increase in apparent frequency of external events as a consequence of internally generated events, then the IFE effect reported in Experiment 1 could not be attributed simply to the "external" component of written recall.

Two additional conditions of testing were investigated. In the "covert image" group, subjects were asked to create a mental picture of the items on test trials. In the "see and image" group, subjects never experienced a true test trial; instead, on trials corresponding to test trials in the other conditions, the subjects were shown the appropriate item for each cue and were asked to create an image of the item. The last two groups were included primarily as a pilot for future work comparing different vs same images. In addition, the covert image group provided some evidence regarding the range of conditions over which the IFE effect is found, and the see and image group provided some information about the effects of additional inputs on judged frequency.

\section{Method}

As in Experiment 1, study and test frequency were manipulated factorially within subjects. The between-subjects variable was the task of the subject on test trials. Cues were presented on test trials in all conditions. The standard recall subjects were tested as in Experiment 1 . Covert recall subjects were instructed to think of the appropriate item and to indicate with a check mark or a zero whether or not they could remember it. Covert image subjects were asked to create a mental picture of the referent of the appropriate word and to rate the vividness of the image on a scale on which 1 indicated an extremely weak 
image and 7 indicated an extremely vivid image. If subjects could not recall the item at all, they indicated this with a zero. In addition, these subjects were asked to make a different mental picture each time a particular item was tested. Subjects in the see and image condition actually saw the correct words along with their cues on "test" trials and were asked simply to create a different image each time the item was tested and to rate the vividness on the same scale as was used in the covert image condition. Thus, see and image subjects never actually had to generate the items themselves.

The procedure was the same as that in Experiment 1 in all important particulars. All subjects were asked to judge the frequency of study opportunities. In addition, immediately after the judgment task, subjects were given a free recall test in which they were to recall as many of the study items as possible. Ten subjects were randomly assigned to each of the four test conditions.

\section{Results}

As before, each subject's data were reduced to a single mean for each study-test cell in the design and these scores were used in the analyses.

Judgments of study frequency. Figure 4 shows the mean judgments of study frequency for each of the four conditions. There was a triple-order interaction of Type of Test by Number of Study Opportunities by Number of Test Trials $[\mathrm{F}(12,144)=2.15, \mathrm{MSe}=.60]$. The triple-order interaction appears to result from variations at different study frequencies in the exact ordering of the test conditions, in terms of the magnitude of the increase in apparent frequency as a consequence of test trials.

The presence of the triple-order interaction does not, however, substantially alter the following conclusions: (1) Estimates of frequency increased with actual increases in the frequency of externally generated events $[\mathrm{F}(2,72)=66.40, \mathrm{MSe}=1.06] ;(2)$ estimates of frequency were increased by test trials $[\mathrm{F}(2,72)=100.99$, $\mathrm{MSe}=.96]$; and (3) as shown in Figure 5, the amount added by test trials depended on the type of test $[\mathrm{F}(6,72)=2.47, \mathrm{MSe}=.96]$. From Figure 5, the amount added to apparent frequency by test trials

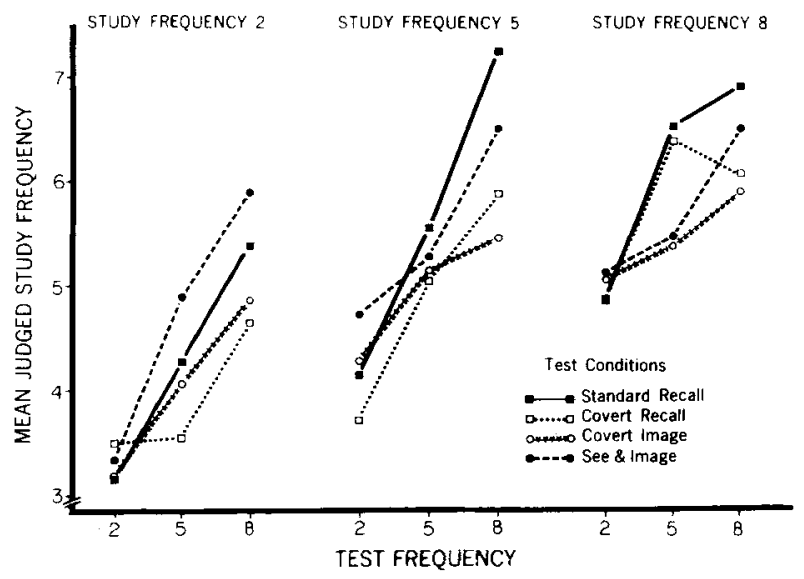

Figure 4. Judged study frequency as a function of test frequency. Each panel represents a different study frequency. Each line represents a different condition (Experiment 2).

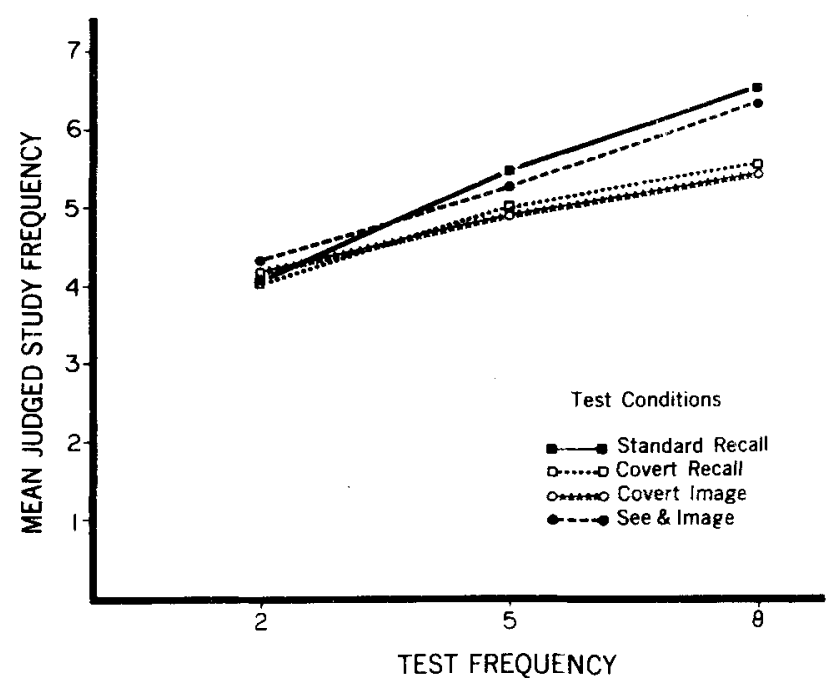

Figure 5. Judged study frequency as a function of manipulated test frequency. Each line represents a different condition (Experiment 2).

was generally greater in the standard recall and see and image conditions than it was in the covert recall and covert image conditions.

Overall, the results indicate that entirely covert, internally generated events can be confused with externally generated events. However, the IFE effect appeared greater with overt recall than with covert recall. In addition, the amount added by overt recall appeared to be no less than that added by additional exposures to the items during which subjects generated and rated images (at least for study frequencies of 5 or 8 ).

Test-Trial Data. Any interpretation of the differences among conditions in the magnitude of the IFE effect depends on whether the differences might be attributed to variations among conditions in the number of "successful" test trials. Therefore, an analysis of test trial performance was conducted.

Scores of the standard recall subjects represent the number of tests on which they produced the correct item; scores for the covert recall subjects represent the number of tests on which they indicated that they could recall an item; scores for the covert image group indicate the number of trials on which subjects indicated they had an image, regardless of quality; and, scores for the see and image condition represent the number of tests on which subjects rated the items (less than perfect scores here essentially indicate tests on which subjects failed for one reason or another to complete the rating task).

For all test conditions combined, the mean number of successful tests were $1.84,4.78$, and 7.70 for Test Frequencies 2,5 , and 8 , respectively $[\mathrm{F}(2,72)=7,910.38, \mathrm{MSe}=.13]$. Thus, subjects' actual frequency of tests was very close to the nominal values of the independent variable. Successful performance on 
Table 1

Mean Number of Successful Test Trials

\begin{tabular}{lccc}
\hline & \multicolumn{3}{c}{ Study Frequency } \\
\cline { 2 - 4 } Test Condition & 2 & 5 & 8 \\
\hline Standard Recall & 4.74 & 4.76 & 4.80 \\
Covert Recall & 4.36 & 4.75 & 4.76 \\
Covert Image & 4.74 & 4.92 & 4.85 \\
See and Image & 4.91 & 4.83 & 4.85 \\
\hline
\end{tabular}

test trials was also related to the number of times an item was studied. The mean number of successful tests for Presentations 2, 5, and 8 were $4.69,4.81$, and 4.81, respectively $[\mathrm{F}(2,72)=6.32, \mathrm{MSe}=.11]$. It is apparent from the means that this is a consequence of a slight advantage for Presentation Values 5 and 8 over Presentation Value 2.

The only other significant effect was a Test Conditions by Number of Presentations interaction $[F(6,72)=3.70, M S e=.11]$. The relevant means can be seen in Table 1. The conditions varied most at Presentation Value 2, where covert recall is substantially below the other conditions and the see and image condition is slightly above. The fact that the see and image condition had more successful tests here may explain why they tended to show the greatest increases as a function of test trials at Presentation Value 2 (see left panel of Figure 4). Likewise, the covert recall condition showed the weakest IFE effect at Presentation Value 2. However, in general, the magnitude of the IFE effect was not related to the small fluctuations in number of successful tests. Therefore, it can be safely concluded that the differences among the conditions summarized in Figure 5 reflect real differences in increases in apparent frequency as a consequence of the type of test trial.

Recall data. After the frequency-of-occurrence judgment task, all subjects were asked to recall as many of the items as possible. The overall mean recall was 21.70. The mean number of items recalled for Presentation Values 2, 5, and 8 were $7.02,7.00$, and 7.68 , respectively $[\mathrm{F}(2,36)=2.91, \mathrm{MSe}=.67, \mathrm{p}<.10]$. The mean recall for standard recall, covert recall, covert image, and see and image conditions was 22.50, 22.00, 23.20 , and 19.10 , respectively $[\mathrm{F}(3,36)=2.32$, $\mathrm{MSe}=1.55, \mathrm{p}<.10]$. In general, recall appeared to be higher when subjects had to generate items on tests (see also Hogan \& Kintsch, 1971). No other main effects or interactions approached significance.

The recall data in combination with the frequency judgments indicate that frequency judgments are more sensitive to manipulations of input and output frequency than is recall. Although Tversky and Kahneman (1973) have suggested that there is no single type of information which is the basis for frequency judgments in all situations, they have demonstrated that one cue may be "assessed availability" (e.g., sensitivity to the strength of the association between an item and the list on which it occurs), and they point out the correlation between recall and the apparent frequency of an item (Leicht, 1968; Underwood et al., 1971). In the present studies, availability (as indexed by the final recall test) was not a very good predictor of frequency judgments. There was a tendency for recall to be higher in the production groups as compared to the see and image group, but the latter condition produced greater increases in apparent frequency of inputs as compared to the covert recall and covert image conditions. These results lend some support to the theoretical separation of the underlying mechanisms on which frequency judgments and recall are based (e.g., Underwood, 1969).

In addition, the fact that the level of recall did not depend on the type of subject-generated event (overt or covert regeneration or images) is also interesting. The present paradigm probably prevents much interitem organization from developing, since each item is treated as independent and tested independently of other items. This was necessary, of course, in order to decrease uncontrolled thoughts during study and to bring regenerations under experimental control during tests. Apparently, when such interitem organization is prevented, variations in instructions such as rehearse vs image have little effect. Thus, the recall results are consistent with interpretations of imagery effects on recall which stress the development of interitem relationships rather than advantages for images per se (e.g., Begg, 1973; Bower, 1972).

\section{DISCUSSION}

Most people have had the experience of not being certain whether they heard or read something, or whether they concocted the notion themselves, and many people have clear "memories" of statements others are sure they did not make, or of childhood events which may not have happened. The present results provide an empirical demonstration of a similar phenomenon, in that subjects confused memory for external presentations and memory for internal regenerations. Each type of event influenced the judged frequency of the other. Understanding the mechanisms which produce confusion between memories for perceptions and memories for imaginations may eventually clarify our understanding of the nature of the representation of each in memory.

It might seem reasonable to suppose that some abstract representation of an event (e.g., a pool of "core" features) is activated whenever the same or similar events occur (either as a consequence of perception or thought). Simply reading out the frequency increments attached to the abstract representation would thus result in the IFE and IFI effects. But, if subjects simply "read out" a single strength or frequency value from the abstract representation, the effect of a presentation or test should have been the same for both judgment conditions. Experiment 1 shows that this was not the case, since test frequency 
affected judgments of test frequency more than it affected judgments of study frequency.

In addition, the above notion does not account for the fact that people can discriminate among occurrences of the same external events (e.g., Hintzman \& Block, 1970, 1971), nor for the fact that most people are fairly successful in the daily task of sorting perception from imagination. Some types of temporal and contextual cues are probably retained in the traces of both external and internal events (e.g., "someone was in the room talking to me and hence he must have made that comment," vs "I was alone, so I must have imagined it"). Furthermore, the mental operations involved in perception and thought are surely different (although they very likely involve some of the same processes, e.g., Atwood, 1971; Brooks, 1967), and memory may include cues to the operations involved in establishing a representation which could serve a discriminative function.

Therefore, as a working hypothesis, the present authors are assuming that there is an abstract representation that includes the core features which an event must have in order to be defined as the same referent as something which has occurred before. Thus, under some circumstances, certain superficial aspects of external occurrences (Hintzman, Block, \& Summers, 1973; Rowe, 1974) or of internal occurrences may not affect frequency judgments. The core representation, then, might have associated with it context-specific frequency counters. Context, as we are using it here, would include whether the occurrence was internally or externally generated (and, if internally generated, whether it was produced overtly), and might also include other information such as temporal or modality cues. By accessing "external" counters when asked to judge study frequency and "internal" counters when asked to judge test frequency, occurrences of the two classes of events could be discriminated. Confusion could result if some "wrong" counters were read during the judgment process. The finding of a greater IFE effect with overt than with covert recall (Experiment 2) would follow from the assumption that similarity of events corresponds to similarity (or proximity) of counters. That is, when judging input frequency, a subject might be more likely to read (in addition to external counters) a nearby internal-with-production counter rather than a more distant internal-withoutproduction counter. A reading based on two (or more) counters showing different frequencies would then result in some compromise judgment between (or among) the totals. The selection of counters to be read and the consequences of the particular selection may vary with experimental manipulations and task requirements. For example, in a standard verbal discrimination task, subjects presumably benefit from using a relatively lax criterion regarding the source of frequency increments (Ekstrand, Wallace, \& Underwood, 1966).
It should be noted that while counter types and similarity among counters have been referred to here for the sake of simplicity, these ideas might alternatively be expressed in terms of the attributes or features which various separate mental traces have in common. Thus, subjects could count or estimate the number of traces having certain features (the core characteristics of the event plus the criterial characteristics for the specific judgment task). In addition, while attention has been focused here on the processes of reading out stored frequency information, it would not be inconsistent to suppose at the same time that the function relating event frequency to stored frequency is different for different types of events.

The general question of the relationship between representations of internal and external events is central to the problem of the mechanisms for memory monitoring. Experiment 1 demonstrated that people are sensitive to the relative frequency of internally generated events, as well as to that of externally generated events. In itself, this is not surprising, but this capacity probably deserves more experimental attention considering its possible functional importance. For example, subjects do not spend equal time studying or rehearsing all items in learning tasks, but, rather, sometimes allocate more time to weak as opposed to strong items (e.g., Zacks, 1969). The relative frequency of internally generated events provides a potential cue for distributing study time. More importantly, individual differences in sensitivity to the relative frequency of internal events, as well as individual differences in susceptibility to the IFE and IFI effects, may be correlated with more or less efficient study patterns.

Certain errors in memory, such as intrusions in recall and false recognitions (e.g., Underwood, 1965), can be interpreted as reflecting failures in monitoring the origins of a memory trace. While the low false-positive rate for an associatively related distractor in recognition may reflect the small percentage of subjects who had that particular implicit associative response at study (Kimble, 1968), it may also testify to the quality of available cues to distinguish internally from externally generated items. Errors are perhaps more easily found with prose material, where subjects often falsely recognize information which was not necessarily implied by the input and which they may have imported during study (e.g., Johnson, Bransford, \& Solomon, 1973; Sulin \& Dooling, 1974). For example, subjects who have heard that "the spy threw the secret document into the fireplace just in time," are likely to say later that the spy burned the secret document. However, one problem with the false recognition paradigm is that it is not always clear that the critical thought occurred prior to the recognition test. It is often equally plausible that, during recognition, subjects made their judgments with whatever information was available at that time, and false positives reflect subjects' best guesses about what 
they heard, based on incomplete evidence. Thus, memory for the gist or structure of the information may lead to errors in recognition or distortions in recall, even when the false information was not previously represented in memory (e.g., Postman, 1954). In order to conclude that the memory representation of an imagination has been confused with the memory representation of a perception, the imagination must have occurred prior to the test. The present work is an initial attempt to gain greater experimental control over the occasion of internally generated events (see also Jacoby, 1974), as well as the occasions on which external events are represented, in order to investigate memory for each.

In summary, a number of current ideas underlying theoretical analyses of memory tacitly or explicitly assume that internal activation of an event is equivalent to external activation of the event. However, it seems reasonable to suppose that memory representations include some cues as to the origins of a representation. While, of course, "thought" is involved in perception and the representation of external events in memory, some meaningful distinction must still be made between internally and externally generated memories. Understanding the differences and similarities in the nature of internally and externally generated memories is important for any theory of the way information is represented in memory. An adequate model must be able to account for both discrimination and confusion between imagination and perception.

\section{REFERENCE NOTE}

1. Johnson, M. K. Constructive aspects of memory: Historical antecedents. Paper presented at the annual meetings of the American Psychological Association, Chicago, September 1975.

\section{REFERENCES}

Atwood, G. An experimental study of visual imagination and memory. Cognitive Psychology, 1971, 2, 290-299.

Battig, W. F., \& Montague, W. E. Category norms for verbal items in 56 categories: A replication and extension of the Connecticut category norms. Journal of Experimental Psychology Monograph, 1969, 80(No. 3, Part 2).

BEGG, I. Imagery and integration in the recall of words. Canadian Journal of Psychology, 1973, 27, 159-167.

Bower, G. H. Mental imagery and associative learning. In L. Gregg (Ed.), Cognition in learning and memory. New York: Wiley, 1972.

Brooks, L. R. The suppression of visualization by reading. Quarterly Journal of Experimental Psychology, 1967, 19, 289-299.
Ekstrand, B. R., Wallace, W. P., \& Underwood, B. J. A frequency theory of verbal-discrimination learning. Psychological Review, 1966, 73, 566-578.

Hintzman, D. L. Apparent frequency as a function of frequency and the spacing of repetitions. Journal of Experimental Psychology, 1969, 80, 139-145.

Hintzman, D. L., \& Block, R. A. Memory judgments and the effects of spacing. Journal of Verbal Learning and Verbal Behavior, 1970, 9, 561-566.

Hintzman, D. L., \& BloCk, R. A. Repetition and memory: Evidence for a multiple-trace hypothesis. Joumal of Experimental Psychology, 1971, 88, 297-306.

Hintzman, D. L., Block, R. A., \& Summers, J. J. Modality tags and memory for repetitions: Locus of the spacing effect. Journal of Verbal Learning and Verbal Behavior. 1973, 12. 229-238.

Hogan, R. M., \& KintsCh, W. Differential effects of study and test trials on long-term recognition and recall. Joumal of Verbal Learning and Verbal Behavior, 1971. 10, 562-567.

Howell, W. C. Representation of frequency in memory. Psychological Bulletin. 1973, 80, 44-53.

JACOBY, L. L. The role of mental contiguity in memory: Registration and retrieval effect. Journal of Verbal Learning and Verbal Behavior, 1974, 13, 483-496.

Johnson, M. K.. BRAnSFord, J. D., \& Solomon, S. K. Memory for tacit implications of sentences. Journal of Experimental Psychology, 1973, 98, 203-205.

KIMBLE, G. A. Mediating associations. Journal of Experimental Psychology, 1968, 76, 263-266.

LEICHT, K. L. Recall and judged frequency of implicitly occurring words. Joumal of Verbal Learning and Verbal Behavior. 1968, 7, 918-923.

Postman, L. Learned principles of organization in memory. Psychological Monographs. 1954. 68(No. 374).

Rowe. E. J. Depth of processing in a frequency judgment task. Joumal of Verbal Learning and Verbal Behavior. 1974. 13, 638-643.

Sulin, R. A., \& Dooling. D. J. Intrusion of a thematic idea in retention of prose. Journal of Experimental Psychology. $1974,103,255-262$.

TVersky, A., \& Kahneman, D. Availability: A heuristic for judging frequency and probability. Cognitive Psychology, 1973, 5, 207-232.

UNDERWOOD, B. J. False recognition produced by implicit verbal responses. Journal of Experimental Psychology, $1965,70,122-129$.

UNDERWOOD, B. J. Attributes of memory. Psychological Review, 1969, 76, 559-573.

UNderW OOD. B. J., Zimmerman, J., \& Freund, J. S. Retention of frequency information with observations on recognition and recall. Journal of Experimental Psychology, 1971, 87. 149-162.

ZACKS, R. T. Invariance of total learning time under different conditions of practice. Journal of Experimental Psychology. 1969. 82, $441-447$

(Received for publication May 27. 1976; revision received September 21.1976.$)$ 